\title{
PREVALENCIA DEL TERCER CONDUCTO EN LA RAÍZ MESIAL DE LOS PRIMEROS MOLARES MANDIBULARES PERMANENTES, EN PACIENTES SOMETIDOS A TRATAMIENTO EN LA CLÍNICA SERODU, ECUADOR
}

\author{
PREVALENCE OF THE THIRD CANAL IN THE MESIAL ROOT OF THE FIRST \\ PERMANENT MANDIBULAR MOLLARS, IN PATIENTS ATTENDED IN SERODU \\ CLINIC, ECUADOR
}

\author{
Alex Chávez-Vega ${ }^{a}$, María Jarrín-Peñafiel a , Olivia Toalombo-Puma ${ }^{a}$, Alex Carrera-Robalino ${ }^{\text {b }}$, Ana Del Carmen \\ Armas $^{\mathrm{cd}}$
}

\section{RESUMEN}

OBJETIVO: Determinar la prevalencia del tercer conducto en la raíz mesial de los primeros molares mandibulares permanentes, de pacientes que acuden por tratamiento de conducto a la Clínica Odontológica de la Universidad Tecnológica Equinoccial, Quito, Ecuador entre enero 2013 y diciembre 2017. MATERIALES Y MÉTODOS: Se presenta un estudio de tipo descriptivo observacional, en una muestra de 55 historias clínicas de pacientes sometidos a tratamiento endodóntico en la Clínica de Atención Odontológica de la Facultad de Ciencias de la Salud "Eugenio Espejo" de la Universidad Tecnológica Equinoccial, entre enero del 2013 y diciembre del 2017. Tras revisión de las historias clínicas de pacientes atendidos en ese período de tiempo y los criterios de inclusión, las radiografías seleccionadas fueron examinadas en negatoscopio con auxilio de una lupa de aumento por una persona entrenada en la lectura de imágenes y con experiencia en tratamientos endodónticos. Los hallazgos fueron reportados en tablas en Excel, específicamente ela boradas para su posterior análisis estadístico mediante el programa SPSS. RESULTADOS: El análisis radiográfico ejecutado determinó que el $25 \%$ de los primeros molares mandibulares presentaron un tercer conducto, en mayor frecuencia en hombres $(p<0,000)$. CONCLUSIÓN: La presencia del tercer conducto en la raíz mesial del primer molar permanente es baja en los pacientes que asisten a la clínica SERODU, pero alta en comparación con lo que han reportado.

Palabras Clave: Anatomía; Endodoncia; Tratamiento del conductor radicular. (Fuente: DeCS BIREME)

\section{ABSTRACT}

Aim: To determine the prevalence of three canals in the mesial root of the first permanent mandibular molars, of patients who come for root canal treatment at the dental clinic of the Equinoctial Technological University, Quito, Ecuador from January 2013 to December 2017. MATERIALS AND METHODS : A descriptive observational study is presented, in a sample of 55 clinical histories of patients with root canal treatment at the dental clinic of the Health Sciences faculty "Eugenio Espejo" of the Equinoctial Technological University, between January 2013 to December 2017. After a review of the medical records of patients treated during this period of time and inclusion criteria, the selected radiographs were examined in a negatoscope with the help of a magnifying glass, by a single person trained in reading images and with experience in endodontic treatments. The findings were reported in specially developed Excel tables, for later statistical analysis, through the SPSS program. RESULTS: The radiographic analysis performed determined that 14 mandibular first molars presented a third canal, more frequently in men $(p<0.000)$. CONCLUSION: The presence of the third canal in the mesial root of the first permanent molar is low, however, the prevalence is higher than in other studies.

Keywords: Anatomy, endodontics, Root canal treatment. (Source: MeSH NLM)

Recibido: 11 de abril 2018

Aprobado: 27 de junio 2018

Publicado: 30 de junio 2018

\footnotetext{
${ }^{1}$ Universidad Tecnológica Equinoccial

a Estudiante

${ }^{\mathrm{c}}$ Odontólogo

${ }^{\mathrm{d}} \mathrm{PhD}$ en Operatoria dental, Docente

Citar como: Chavez -Vega A, Jarrín-Peñafiel M, Toalombo-Puma O, Carrera-Robalino A, Armas Vega A. Prevalencia del tercer conducto en la raíz mesial de los primeros molares mandibulares permanentes, en pacientes sometidos a tratamiento en la clínica SERODU, Ecuador. KIRU. 2018; 15(2):63-68. https://doi.org/10.24265/kiru.2018.v15n2.01 


\section{INTRODUCCIÓN}

El éxito en el tratamiento endodóntico constituye el conocimiento de la anatomía del conducto radicular ${ }^{(1)}$ asociada a la limpieza mecánica y química del sistema radicular estableciendo los objetivos de la endodoncia(2). La morfología considerada normal nos muestra que en el $59 \%$ de los casos existen dos canales radiculares en el conducto mesial, confluyentes entre sí en el $28 \%{ }^{(3)}$, sin descartar que en este puede encontrarse un tercer conducto mesial $(1 \%)$ y en escasas ocasiones unión de ellos formando un plexo ${ }^{(4)}$, que desembocan por separado o con cierta frecuencia pueden unirse a uno de los canales principales $(38 \%)^{(5)}$. Así mismo, la presencia de dos conductos en la raíz distal se ha visto en el $15 \%$ de los casos (3); también puede ser encontrada, mostrándose generalmente muy amplio y bifurcado en el ápice ${ }^{(6)}$.

Esta discrepancia en cuanto a su anatomía explicaría ciertos fracasos observados cuando este diente es tratado endodónticamente ${ }^{(7)}$.

Considerando que el primer molar inferior es, sin duda, junto al primer molar maxilar, el diente que más tratamientos de conductos precisa ${ }^{(8)}$, resulta importante durante el tratamiento endodóntico localizar todos y cada uno de los conductos que forman parte del entramado radicular ${ }^{(4)}$.

Dentro de la formación de un estudiante universitario de Odontología, la elaboración de tratamientos endodónticos constituye un requisito imprescindible. Debido al reporte de la necesidad de un incremento en el número de citas para tratamiento de primeros molares definitivos, se decide mediante este estudio establecer la prevalencia del tercer conducto en la raíz mesial de molares mandibulares permanentes, planteándose ejecutar una revisión visual de radiografías periapicales de pacientes que acuden a la Clínica de Atención Odontológica de la Universidad Tecnológica Equinoccial (UTE), Quito, Ecuador, en el período de enero 2013 a diciembre 2017.

\section{MATERIALES Y MÉTODO}

Se plantea un estudio del tipo descriptivo observacional, ejecutado con la autorización de las autoridades de la Clínica SERODU, donde fueron evaluadas historias clínicas de pacientes sometidos a tratamiento de conducto en la clínica durante el periodo de enero 2013 a diciembre 2017. Cada una de las historias clínicas fue examinada, escogiendo únicamente aquellas que describieron haber recibido tratamiento de conducto de los dientes 3.6 y/o 4.6, que contaran con radiografías periapicales con imágenes claras, y que la edad del paciente se encontrara entre los 18 y 70 años.

Tras esta selección, las radiografías seleccionadas fueron examinadas mediante un negatoscopio con auxilio de una lupa de aumento en busca de un tercer canal, acción que fue ejecutada por una única persona entrenada en la lectura de imágenes y con experiencia en tratamientos endodónticos. Los hallazgos fueron verificados en la historia clínica de cada paciente y reportados en tablas en Excel específicamente elaboradas.

Estos datos fueron analizados mediante el programa SPSS a través de un análisis estadístico de correlación para la verificación de relación o no entre nuestras variables.

\section{RESULTADOS}

La evaluación preliminar arrojó 355 historias clínicas de pacientes sometidos a tratamiento de conducto en la Clínica de Atención Odontológica de la UTE en el periodo de enero 2013 a diciembre 2017, de los cuales 55 historias clínicas relataban la ejecución de tratamiento endodóntico del diente 3.6 y/o 4.6. El examen visual de las radiografías periapicales permitió verificar que 14 radiografías mostraron la presencia de un tercer conducto en la raíz mesial, correspondiendo a un $25 \%$ de la muestra examinada; de los cuales el $35,7 \%$ pertenecieron a pacientes de género femenino y el $64,3 \%$ restante al sexo masculino, con una relevancia estadísticamente significativa entre el sexo y la presencia de un tercer conducto $(p<0,002)$. Al respecto, sobre la pieza, $57 \%$ pertenecieron al diente 4.6 y el $43 \%$ al diente 3.6 .

Después del análisis estadístico se obtuvo una significancia estadísticamente significativa entre la pieza y la presencia de un tercer conducto $(p<0,000)$.

\section{DISCUSIÓN}

Nuestros resultados mostraron la presencia de un tercer conducto en la raíz mesial de los dientes 3.6 y 4.6 en un $25 \%$, teniendo una mayor prevalencia que estudios realizados anteriormente. Vertucci, en 1984, encontró solo el $1 \%$ de tres conductos en la raíz mesial $^{(3)}$. Wolf obtuvo el $0,8 \%{ }^{(9)}$. Forne, en su estudio in vitro obtuvo el $14,81 \%$ de tercer conducto mesial en 27 dientes analizados, empleando tomografía computarizada, y el $12 \%$ en 25 dientes evaluados empleando microscopio electrónico de barrido ${ }^{(6)}$. Esta baja prevalencia también fue determinada en revisión sistemática realizada por Bentacour y colaboradores, considerando 41 estudios que 
incluían un total de 18.781 dientes, en los que la presencia de esta alteración morfológica alcanzó el $13 \%$ y se correlacionó fuertemente con la etnia de la población estudiada ${ }^{(4)}$.

Lu $Q$ y cols, refiere dos casos en la población china, en un hombre de 28 años con dolor severo en primer molar izquierdo mandibular, con diagnóstico de necrosis pulpar y con sombra radiolúcida compatible de periodontitis periapical crónica, el acceso permitió observar los canales MB, ML, DV, DL que únicamente tras limpieza y conformación fue detectado con el DG-16 un orifico entre MB-ML correspondiente al conducto $\mathrm{MM}$; un caso semejante en mujer de 55 años remitida para el tratamiento endodóntico del primer molar derecho mandibular con pulpitis irreversible, mostro tras el acceso tres canales hacia mesial y dos hacia distal, según los autores la incidencia de MM reportada en la literatura si bien es baja (2.07\% a 15\%) exige del clínico operador utilizar todos los medios de diagnósticos adecuados durante la preparación del conducto ML. (10).

Por otro lado, la frecuencia de esta alteración anatómica aumentó al 69,82\% y de forma más frecuente en mujeres cuando fueron analizadas quinientas cincuenta imágenes, observando, asimismo, una relación directa con la etnia ${ }^{(11)}$. Así entonces, considerando que el ecuatoriano es un individuo resultante de la mezcla de genes indígenas, españoles y negros ${ }^{(12)}$, estarían justificados estos resultados (13). En estudios anatómicos de los conductos realizados en otros continentes, como el de Muriithi en la población keniata, este obtuvo el $0,5 \%$ de prevalencia, muy baja en comparación con lo obtenido ${ }^{(14)}$. Nosrat encontró $20 \%$ de las piezas analizadas con tres conductos mesiales, lo que se asemeja con el $25 \%$ encontrado en nuestra población (15)

$\mathrm{Si}$ bien la muestra en nuestro estudio fue relativamente baja, los resultados abren nuevas interrogantes y deseos de relacionar estos hallazgos con un estudio genético de la muestra, lo cual permitiría obtener datos confiables para la toma de decisiones en cuanto al tratamiento endodóntico y su relación con el conocimiento de la anatomía radicular (13), buscando siempre prevenir el fracaso $\left({ }^{16)}\right.$, siempre acompañado de la experiencia clínica y del empleo de localizadores, radiografías, magnificación y ultrasonidos ${ }^{(17)}$, siendo este una de los primeros estudios sobre anatomía interna en el país.
Una de las limitaciones más importantes en el estudio fue el hecho de contar solamente como fuente de información con la historia clínica y las radiografías reportadas en ella, lo que impidió disponer de un consentimiento informado por parte de cada paciente y obtener información sobre su origen o etnia e incluso la toma de muestras de sangre, lo que resultaría extremadamente provechoso para realizar un análisis genético y asociar la presencia de estas alteraciones morfológicas y la identificación genética, sobre todo buscando aprovechar estos conocimientos en los protocolos endodónticos a realizarse ${ }^{(18)}$, entendiendo que la adaptación a estas modificaciones desde sus etapas de formación resulta básica e imprescindible ${ }^{(19)}$.

Buscamos, desde la academia como ente formador de nuevos profesionales, tratar estos aspectos en los microcurrículos de las asignaturas correspondientes, reconociendo siempre la importancia del empleo de nuevas tecnologías de diagnóstico y tratamiento actuales (20) así como de un entrenamiento en la preparación de estas anomalías para poder superar estos retos de forma adecuada (21). Consideramos, asimismo, que los resultados y la prevalencia de esta alteración anatómica en la población estudiada, los tiempos y protocolos de tratamiento requerido deben ser respetados.

Como consecuencia de la ejecución de este estudio se puede concluir que existe una prevalencia considerable $(25 \%)$ de tres conductos en la raíz mesial de los primeros molares mandibulares de pacientes entre 12 y 70 años que acuden a la Clínica de Atención Odontológica de la UTE, Quito, Ecuador, entre enero del 2013 y diciembre del 2017.

Participación de autoría: ACR y ACA participaron en el diseño del estudio. ACV,MJP y OTP recolectaron y procesaron los datos. ACR y ACA realizaron la revisión crítica del artículo. Todos los autores aprobaron la versión final del manuscrito.

Conflicto de interés. Los autores manifestaron no tener conflictos de interés

Fuente de financiamiento: Autofinanciado.

\section{REFERENCIAS BIBLIOGRÁFICAS}

1. Rouhani A, Bagherpour A, Akbari M, Azizi M, Nejat A, Naghavi N. Cone-beam computed tomography evaluation of maxillary first and second molars in Iranian population: a morphological study. Iran Endod, $2014 \mathrm{~J} ;$ 9(3): 190-194.

2. Pereira R, Pereira G, Barroso J, Barroso C, Bortolotti $M$, Junqueira J. Effect of $17 \%$ EDTA on removal of canal wall smear layer and calcium hydroxide 
dressing: Part II. RGO - Rev Gaúcha Odontol; 2014 62(1): 53-8.

3. Vertucci F. Root canal morphology and its relationship to endodotic procedures. Endodontic Topics. 2005, 10, 3-29.

4. Betancourt $P$, Cantin M, Fuentes R. Frecuencia del canal MB2 en la raíz mesiovestibular del primer molar maxilar en estudios in vitro e in vivo: una revisión sistemática. Av Odontoestomatol. 2014; 30(1): 11-22.

5. Betancourt P, Fuentes R, Aracena Rojas S, Cantín M, Navarro Cáceres P. Configuración anatómica del sistema canalicular de la raíz mesiovestibular del primer molar maxilar. Av Odontoestomatol. 2015; 31(1): 11-18.

6. Forner-Navarro L, Luzi A, Almenar-García A, HervásGarcía A. Third canal in the mesial root of permanent mandibular first molars: review of the literature and presentation of 3 clinical reports and 2 in vitro studies. Med Oral Patol Oral Cir Bucal. 2008 Dec 1; 12(8): E605-9.

7. Choi D, Ga Yeun L, Choi A, Torabinejad M. Difference in the incidence of the second mesiobuccal canal between Caucasian and Malaysian maxillary first molars. ENDO (Endodontic Practice Today). Fall 2015; 9(3): 169-175.

8. Betancourt $P$, Navarro $P$, Muñoz $G$, Fuentes $R$. Prevalence and location of the secondary mesiobuccal canal in 1,100 maxillary molars using cone beam computed tomography. BMC Medical Imaging. 2016; (16)66.

9. Wolf TG, Paqué F, Zeller M, Willershausen B, BriseñoMarroquín B. Root Canal Morphology and Configuration of 118 Mandibular First Molars by Means of Micro-Computed Tomography: An Ex Vivo Study. J Endod. 2016 Apr; 42(4): 610-4.

10. Lu Q, Wang $P$, Yang B, Liu XJ, Wang SY, Yu Q. Endodontic treatments of mandibular first molar with middle mesial canal: two case reports. Chin J Dent Res. 2013;16(1):75-8. PubMed PMID: 23878830.

11. De Pablo OV, Estevez R, Péix Sánchez M, Heilborn C, Cohenca N. Root anatomy and canal configuration of the permanent mandibular first molar: a systematic review. J Endod. 2010 Dec; 36(12): 191931.

12. Valencia O, Esteveza R, Heilbornb C, Cohenca N. Anatomía radicular y configuración de conductos del primer molar inferior permanente. Quintessence (ed. esp.). 2012; 25: 538-49.
13. Tinelli M, Ferreira G, Gómez N, Llahyah K. Variantes étnicas de la topografía de los conductos radiculares. Electronic Journal of Endodontics (Rosario) [Internet] 2011. [Citado: 23 de diciembre 2017]; Vol 2: 553-557. En: https://dialnet.unirioja.es/descarga/articulo/3740656.p df

14. Muriithi NJ, Maina SW, Okoth J, Gathece LW. Internal root morphology in mandibular first permanent molars in a kenyan population. East Afr Med J. 2012 May; 89(5): 166-71.

15. Nosrat A, Deschenes RJ, Tordik PA, Hicks ML, Fouad AF. Middle mesial canals in mandibular molars: incidence and related factors. J Endod. 2015 Jan; 41(1): 28-32.

16. Rouhani A, Bagherpour A, Akbari M, Azizi M, Nejat A, Naghavi N. Cone-beam computed tomography evaluation of maxillary first and second molars in Iranian population: a morphological study. Iran Endod, 2014 J. 9(3): 190-194.

17. Álvarez J, Clavera T, Becerra O, Rodríguez Y, Boris E. Tratamiento endodóntico radical en pulpa no vital en una sola visita. Rev haban cienc méd. 2014; 13(2): 219226.

18. Matta MS, Kaur M. Endodontic management of middle mesial canal in mandibular first molar. Ann Trop Med Public Health [Internet]. 2015 [citado: Abril 3 de 2018]; 8:60-3. Disponible en: http://www.atmph.org/text.asp?2015/8/3/60/157631

19. Huilcarema AU. Tesis: Estudio in vitro del incidencia del conducto mesio medial del primer molar inferior en una muestra de mil piezas extraídas. [Internet].; 2015 [citado: Abril 3 de 2015. Disponible en: http://repositorio.ug.edu.ec/bitstream/redug/9032/1/U RGILESalberto.pdf

20. Pattanshetti N, Gaidhane M, Kandari A. Root and canal morphology of the mesiobuccal and distal roots of permanent first molars in a Kuwait population - a clinical study. Int Endod J 2013; 41: 755-62.

21. Hosoya N, Yoshida $T$, lino $F$, Arai $T$, Mishima $A$, Kobayashi K. Detection of a secondary mesio-buccal canal in maxillary first molar: A comparative study. J Conserv Dent. 2013; 15: 127-31. 